\title{
The nature and nurture of cell heterogeneity: accounting for macrophage gene-environment interactions with single-cell RNA-Seq
}

Quin F. Wills ${ }^{1,2^{*}+}$, Esther Mellado-Gomez ${ }^{1 \dagger}$, Rory Nolan ${ }^{1,3}$, Damien Warner ${ }^{4}$, Eshita Sharma ${ }^{1}$, John Broxholme ${ }^{1}$, Benjamin Wright ${ }^{1}$, Helen Lockstone ${ }^{1}$, William James ${ }^{4}$, Mark Lynch ${ }^{5}$, Michael Gonzales ${ }^{5}$, Jay West ${ }^{5}$, Anne Leyrat ${ }^{5}$, Sergi Padilla-Parra ${ }^{1,3}$, Sarah Filippi ${ }^{6}$, Chris Holmes ${ }^{1,6}$, Michael D. Moore ${ }^{4^{*}}$ and Rory Bowden ${ }^{1 *}$

\begin{abstract}
Background: Single-cell RNA-Seq can be a valuable and unbiased tool to dissect cellular heterogeneity, despite the transcriptome's limitations in describing higher functional phenotypes and protein events. Perhaps the most important shortfall with transcriptomic 'snapshots' of cell populations is that they risk being descriptive, only cataloging heterogeneity at one point in time, and without microenvironmental context. Studying the genetic ('nature') and environmental ('nurture') modifiers of heterogeneity, and how cell population dynamics unfold over time in response to these modifiers is key when studying highly plastic cells such as macrophages.

Results: We introduce the programmable Polaris ${ }^{\mathrm{TM}}$ microfluidic lab-on-chip for single-cell sequencing, which performs live-cell imaging while controlling for the culture microenvironment of each cell. Using gene-edited macrophages we demonstrate how previously unappreciated knockout effects of SAMHD1, such as an altered oxidative stress response, have a large paracrine signaling component. Furthermore, we demonstrate single-cell pathway enrichments for cell cycle arrest and APOBEC3G degradation, both associated with the oxidative stress response and altered proteostasis. Interestingly, SAMHD1 and APOBEC3G are both HIV-1 inhibitors ('restriction factors'), with no known co-regulation.

Conclusion: As single-cell methods continue to mature, so will the ability to move beyond simple 'snapshots' of cell populations towards studying the determinants of population dynamics. By combining single-cell culture, live-cell imaging, and single-cell sequencing, we have demonstrated the ability to study cell phenotypes and microenvironmental influences. It's these microenvironmental components - ignored by standard single-cell workflows - that likely determine how macrophages, for example, react to inflammation and form treatment resistant HIV reservoirs.
\end{abstract}

Keywords: Single-cell sequencing, Single-cell culture, Single-cell imaging, Macrophage heterogeneity, Signaling microenvironment

\footnotetext{
*Correspondence: qilin@well.ox.ac.uk; kenny.moore@path.ox.ac.uk;

rbowden@well.ox.ac.uk

${ }^{\dagger}$ Equal contributors

'Wellcome Trust Centre for Human Genetics (WTCHG), University of Oxford,

Oxford OX3 7BN, UK

${ }^{4}$ Sir William Dunn School of Pathology, University of Oxford, Oxford OX1 3RE,

UK

Full list of author information is available at the end of the article
}

(c) The Author(s). 2017 Open Access This article is distributed under the terms of the Creative Commons Attribution 4.0 International License (http://creativecommons.org/licenses/by/4.0/, which permits unrestricted use, distribution, and reproduction in any medium, provided you give appropriate credit to the original author(s) and the source, provide a link to the Creative Commons license, and indicate if changes were made. The Creative Commons Public Domain Dedication waiver (http://creativecommons.org/publicdomain/zero/1.0/) applies to the data made available in this article, unless otherwise stated. 


\section{Background}

Macrophages - cells that phagocytose microbes, unhealthy and cancerous cells - are at the heart of human aging and pathology from infectious and noninfectious aetiologies. As immune sentinels, macrophages exhibit a variety of pro- and anti-inflammatory phenotypes. At the cellular level these phenotypes are determined not only by (epi-)genetic lineage but are also highly plastic to changing tissue environments [1]. The interplay between these phenotypic drivers underlies many macrophage-mediated pathologies. For example, the complex infectious dynamics between HIV-1 and macrophages within particular tissue niches not only prevents virus eradication in patients on antiretrovirals [2], but is a likely source of low grade neuroinflammation leading to neurocognitive decline [3]. Gene-edited macrophages can be used to study genes with known host-pathogen interactions, but in the absence of genetically tractable blood derived macrophages, macrophages derived from genetically modified pluripotent stem cells (PSCs) provide a suitable alternative model system. These stem cell models have the advantage of reproducibly producing large numbers of edited cells under controlled conditions $[4,5]$. However, one challenge with stem cell models is the intersection of biological and technical (stem cell differentiation) heterogeneity that needs to be accounted for, making the case for single-cell sequencing. In addition, with such highly plastic cells it is important to be able to study the context of genetic modifiers, by controlling signaling microenvironments and cell interactions. It's this context that is crucially lost with many single-cell sequencing approaches, and so a technical goal would be to be able to include the effects of multiple environmental, signaling and intervention variables on cell population phenotypes and dynamics.

To allow for a genotype-by-environment investigation we cultured over 500 CRISPR-edited macrophages using a novel microfluidic platform that allows time and dose control over each individual cell's microenvironment [6] (Fig. 1a). A mixture of wild-type and SAMHD1 knockout monocytes, were generated from HUES-2 human embryonic stem cells [4]. These were differentiated into macrophages that resemble blood monocyte derived macrophages, both phenotypically (high phagocytic ability, expression of CD14, HIV-1 infectability) and transcriptomically [7], while sharing ontogeny with specific tissueresident macrophages such as microglia of the central nervous system $[8,9]$. The need for and importance of such tissue-resident models has recently been reviewed by Sattentau and Stevenson [10]. As the protein of interest in these cells, SAMHD1 is a poorly understood dNTPase that has emerged as a potent HIV-1 restriction factor in non-cycling cells [11]. Its primary physiological role is believed to be the maintenance of genome integrity by limiting the dNTP pool when DNA replication is not required, which is in keeping with observed SAMHD1 downregulation in several cancers $[12,13]$. Of direct relevance to innate immunity is its congenital loss of function associated with Aicardi-Goutieres syndrome, a neurodegenerative disease linked to dysregulated inflammation [14]. In order to gain better insights into SAMHD1 biology within macrophages, we set out to study the knockout of this gene in our model. RNA sequencing at a single-cell resolution was necessary to, at a minimum, rule out technical contributions from unwanted cell populations that can occur with imperfect stem cell model differentiation. Furthermore, as SAMHD1 has a direct association with inflammation signaling, we sought to do this in a way that would not only study aspects of inflammatory activation but also cell autonomous effects separate from the influences of macrophage paracrine signaling.

Per microfluidic chip, up to 48 individual macrophages were isolated at random from a mixture of differentially stained wild-type and knockout cells, and cultured in isolation under different inflammatory signaling environments. These culture environments comprised four possible combinations of exposure or non-exposure to lipopolysaccharide (LPS, to stimulate inflammatory activation), and standard or conditioned media. Conditioned medium was derived from bulk macrophage cultures, to simulate the intercellular signaling component that is important in the control of macrophage inflammatory activation. In total this provided eight cell populations per chip (each cell having one of two genetic conditions, and one of four culture environment conditions), with each chip cultured for either one or eight hours in order to account for early temporal changes in response. Details of the experimental work flow are provided in Additional file 1: Figure S2. Cellular phenotypes such as motility and morphology were tracked by live-cell imaging, before the cDNA from cells was harvested for single-cell RNA sequencing. All of the aggregate 16-fold combinations of genotype, environment and time were repeated in at least nine replicate runs to ensure robustness of conclusions. Details of the experimental and imaging methods, including the sequencing quality control are provided in the Methods and Additional file 1.

\section{Results}

Identifying different macrophage states (phenotypes) Exploratory RNA-seq data analysis (Fig. 1b) revealed a clear differentiation between one- and eight-hour cultured cells. It also highlighted a subgroup of cells present at both time points that enriched for a gene set previously demonstrated to be differentially expressed in macrophages treated with the (anti-)inflammatory TGFB cytokine (Additional file 1). This provided initial 


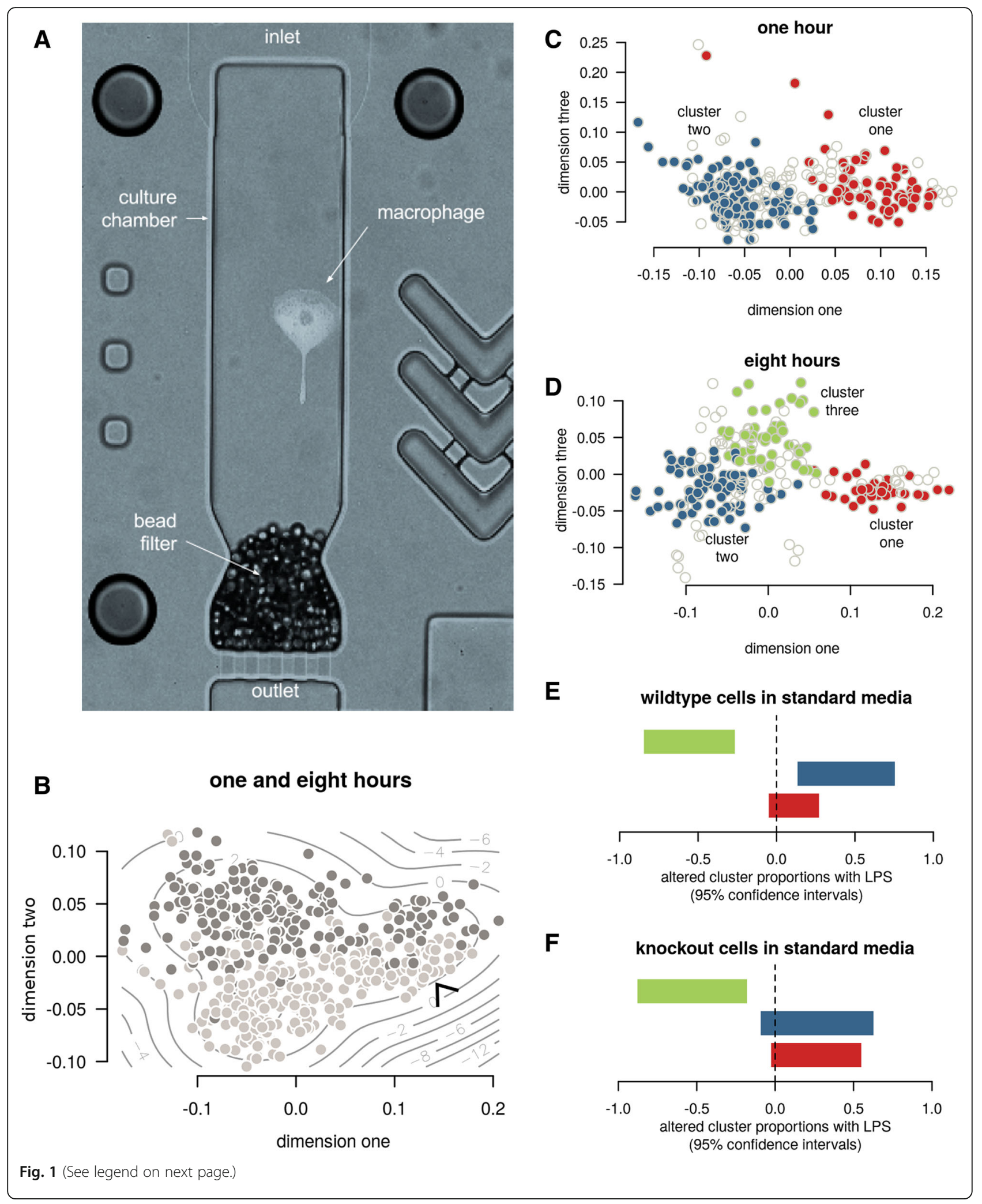


(See figure on previous page.)

Fig. 1 Macrophage culture and subtypes. a A single micro-volume culture chamber from the Polaris ${ }^{\mathrm{TM}}$ microfluidics chip, containing a macrophage. The media conditions per chamber can be modified to study microenvironmental perturbations. $\mathbf{b}$ Visualization of the major cell differences — such as with this multi-dimensional scaling of the transcriptomic rank correlations - demonstrated the separation of cells cultured for one hour (light), eight hours (dark), and a reproducible subcluster present across both time points (arrow). c\&d Formal clustering confirmed this subcluster (cluster one) in addition to a third subcluster emerging after eight hours. The inner 50\% of cells in each cluster are shown in colour for dimensions one and three to better convey relative cluster positions and densities. e\&f Cluster three significantly reduced its proportion in the context of LPS and standard media. 95\% confidence intervals for change in proportions are shown

evidence for the potential biological relevance of the observed heterogeneity among the macrophages. To more formally study this latent/emergent (i.e. previously unknown) heterogeneity and the gene expression differences underlying it, we developed a novel hybrid model-driven and non-parametric clustering method constrained to report only cell clusters that were well represented across replicates. We provide further details in the Methods, with all analysis code and results provided in the Additional files 1 and 2 respectively. Our more formal clustering analysis confirmed the existence of the lower-abundance cell state at both time points (called 'cluster one' in Fig. 1c\&d) but also a reproducible third state (called 'cluster three') emerging after eight hours. A search for associations between proportions of the cell states and culture conditions revealed a lower abundance of cluster three when in standard media with LPS (Fisher's Exact $-\log _{10} \mathrm{P}=5.14$, Fig. 1e\&f), suggesting another inflammatory subphenotype in the cells. With these results demonstrating heterogeneity that would otherwise be missed with traditional sequencing, we then asked if any of these cell types resembled the tissue-resident phenotype of interest.

\section{Identifying the macrophage phenotype of interest}

To better understand these latent cell states, we used our modification on the Heskes Rank Product method [15] to estimate upper and lower p-value bounds on differential and heterogeneous gene expression. As detailed in the Methods, we defined differential expression as a global/overall shift in gene expression, while heterogeneous/context-specific expression was defined as highly variable expression across cell states or culture microenvironments. We selected this non-parametric gene ranking approach not only for its focus on result reproducibility, but for its speed and ease of data fusion that make it well suited to single-cell analysis.

Compared against one-hour cultures, most cells in eight-hour cultures (cluster two cells) progressed towards an anti-inflammatory transcriptional signature consistent with the tissue-resident macrophage phenotype of interest. This is evidenced, for example, by the expression of IL1RN, encoding IL-1 receptor antagonist, which increased 18-fold over time in cluster two (Fig. 2a). Recombinant IL1RN is used to treat severe inflammatory conditions mediated by its ligand, the archetypal pro- inflammatory cytokine IL-1 [16]. In contrast to other cells, cluster one cells did not move towards a transcriptional profile consistent with a tissue-resident phenotype, and tended to maintain up- or down-regulation of clusterspecific genes. This pattern included lower expression of GAPDH and TPI1, genes involved in glycolysis, a pathway known to vary across macrophage activation phenotypes [17]. Compared with the other clusters, cluster one expressed higher levels of FOXP1 across all culture conditions (Fig. $2 \mathrm{~d},-\log _{10} \mathrm{P}$ of 6.03 and 4.85 at one and eight hours, both globally significant at a 5\% FDR). FOXP1 is a transcription factor involved in maintaining embryonic stem cell pluripotency that must be turned off for complete monocyte differentiation into macrophages [18]. Concerned that selective expression of FOXP1 might represent technical differentiation heterogeneity, we used the imaging data to search for other contributing factors. While we did not find evidence for cell motility or morphology associations, we noted that cluster one cells were more likely to have come into contact with the culture chamber retention beads used to prevent cells from escaping (Fig. 1a, Fisher's Exact $-\log _{10} \mathrm{P}=4.10$ ). We also noted that cells cultured towards the edges of the chips were more likely to touch retention beads (Fisher's Exact $-\log _{10} \mathrm{P}=1.79$ ), but that the chip edge positions were enriched for in cluster one, seemingly independent of the bead association (Fisher's Exact $-\log _{10} \mathrm{P}=3.19$, Additional file 1: Figure S6). As cell imaging was performed hourly, this may in part reflect false negatives for detecting bead contact of cells in a less mature and adherent state. Notwithstanding the evidence for both differentiation and environmental factors underlying cluster one, the low correlation between cluster one and off-chip bulk tissue samples (Additional file 1: Figure S7) led us to conclude that it may represent a potentially interesting but lowabundance phenotype that is unrelated to our main question of SAMHD1 biology in tissue-resident macrophages.

A comparison of gene expression in cluster three versus other cells (Fig. 2b) was consistent with a shift towards macrophages with a tissue remodelling phenotype. This was evidenced, for example, by greater expression of TIMP3, an inhibitor of extracellular matrix degradation, and $C H I 3 L 1$, a secreted glycoprotein also thought to drive tissue remodelling and a known genetic risk factor for asthma severity [19]. Since cluster three's transcriptional 


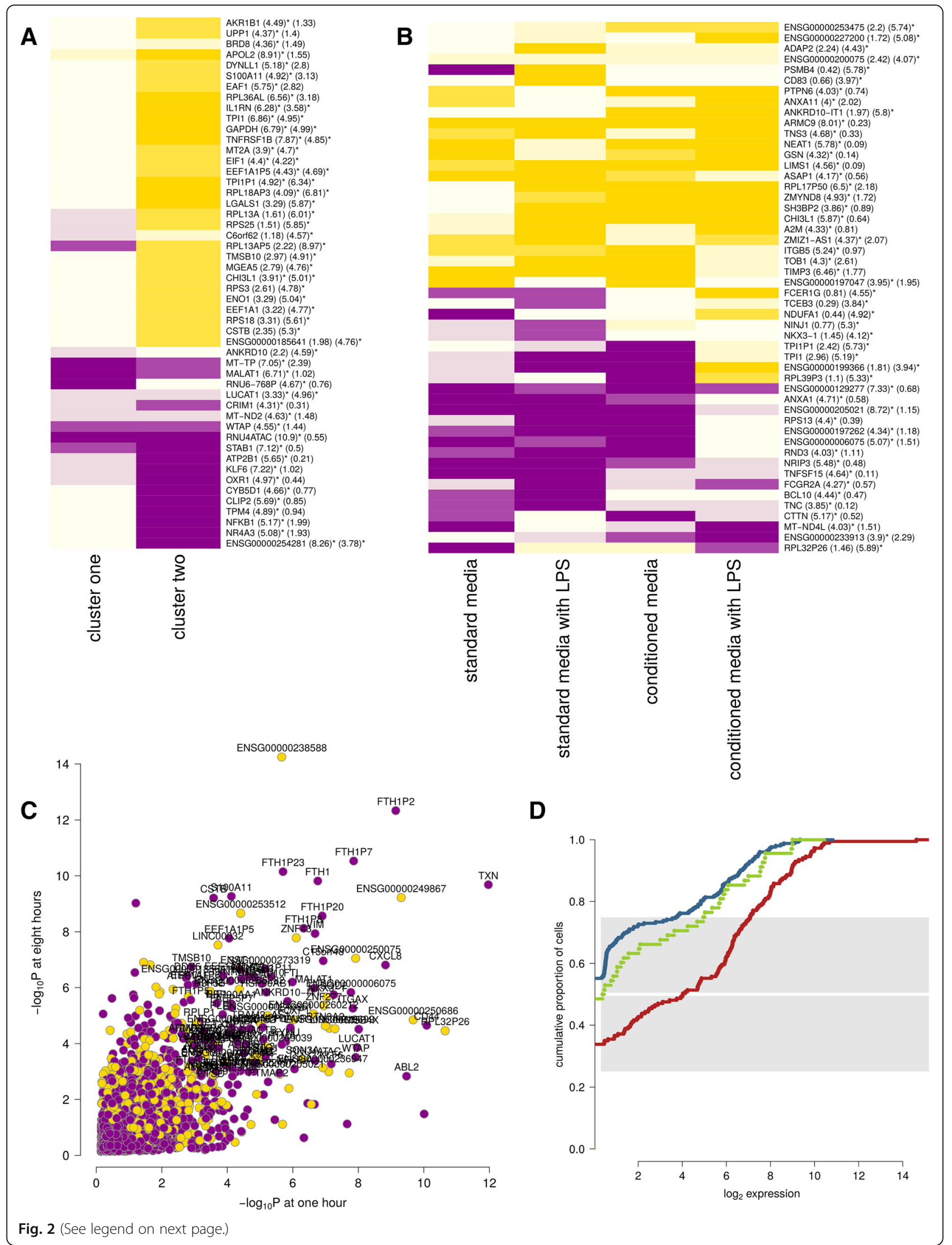


(See figure on previous page.)

Fig. 2 Cell cluster gene expression. In each plot, yellow indicates increased and magenta indicates reduced gene expression. a-b Heatmaps of the top 50 gene expression results, ranked by statistical significance, are shown for clusters one and two over time (a) and cluster three versus other cells, broken down by culture condition (b). The numbers provided in parentheses in this and other heatmaps are -log 10 p-values for differential and heterogeneous (context specific) expression respectively. Results that are globally significant after $5 \%$ false discovery rate (FDR) correction are marked with an asterisk. c The differential expression results for cluster one versus other cells at one and eight hours. $\mathbf{d}$ A cumulative proportion plot for FOXP1 expression broken down by cell clusters. As in other plots, clusters one, two and three are plotted in red, blue and green respectively. Each line plots the cumulative proportion of cells at or below a certain expression level. Cluster one demonstrates greater expression, with approximately half of cluster two and three cells having no detectable expression

phenotype most closely resembles that of cluster two (Additional file 1: Figure S18), it seems plausible that this represents an emerging secondary phenotype that requires macrophage signaling when cells are exposed to LPS (Fig. 1e\&f). Future studies over longer culture periods will help clarify the microenvironmental dependence and persistence of this cluster.

We focus the remainder of our observations on the main body of cells (cluster two), resembling the desired tissueresident phenotype. While no cluster two enrichment for particular motility or morphology imaging features were found over these short culture periods, we briefly note an imaging subtype of cells observed to be phagocytosing the retention beads. Phagocytosing macrophages did not assume a distinct transcriptomic cluster - likely due to short culture times - nevertheless they did enrich for oxidative stress and mitochondrial genes, examples being PRDX1 and MT-CO3 (- $\log _{10} \mathrm{P}$ of 4.60 and 4.43 respectively, both globally significant at $5 \%$ FDR). This unexpected result suggests an avenue for single-cell studies to explore the temporal dynamics of phagocytosis [20].

\section{Changes in macrophage behavior (cluster two) with SAMHD1 knockout}

After filtering our data to focus on the cell subtype of interest (cluster two), we tested for varying knockout and wild-type differential and heterogeneous expression over time. The most striking feature of the globally significant knockout effects was that they were predominantly microenvironment specific (Fig. 3a). Not only does this stress the importance of studying gene-environment interactions in cellular genetics models with known phenotypic plasticity, but in this work allows us to comment on macrophage signaling contributions. Specifically, we note a highly significant association with SOD1 expression, a gene that encodes the cytosolic isozyme of superoxide dismutase. SOD1 is a superoxide radical scavenger that may confer some protection against HIV1 neuropathy as part of the oxidative stress response [21], but which has no described associations with HIV1 restriction factors. As with SAMHD1 loss of function, gain-of-function mutations in SOD1 are associated with neuroinflammation and degeneration, clinically manifesting as amyotrophic lateral sclerosis, which is likely a result of toxic protein aggregates [22]. Overall, SOD1 transcript levels in this study were similar in wild-type and SAMHD1 knockout cells. However, whereas SOD1 expression in wild-type cells stimulated with LPS increased over time as expected, expression in knockout cells increased over time in the absence of LPS activation. Not only does this point to a qualitative difference in expression but - as can be seen from the barely detectable expression changes in standard media (Fig. 3a) - the magnitude of the difference is much greater in conditioned media. This SOD1 oxidative stress response thus has both a notable genetic and signaling component, which may be dampened by identifying and blocking the augmenting signaling factor(s).

Further exploring this oxidative stress response, we noticed a change in SOD1's coexpression (i.e. genes correlating with it) in knockouts versus wild-types (Fig. 3c). Analysing which genes tend to coexpress with SOD1 allows for biological contextualisation of this effect, as genes are only expressed when required, and so expression correlation implies coregulation. A key difference here being the increased coexpression resolution offered by single-cell analysis to generate gene networks in both perturbed and unperturbed cells, compared with conventional sequencing that is limited to only studying average gene correlations with perturbation. We asked whether there are genes with which SOD1 alters its rank (Spearman) correlation across cells when SAMHD1 is knocked out. Fig. 3b lists the globally significant signatures from MSigDB v5.1 [23], consisting of gene sets more correlated with $S O D 1$ in knockout versus wildtype cells. In other words, these highlight genes and pathways coregulating with the altered oxidative stress response in the knockout macrophages. In addition to the expected enrichment for reactive oxygen species (ROS) modulators in this list, we note a striking enrichment for proteasome genes. The proteosome is known to be activated and dysregulated with SOD1 mutation, leading to aberrant cellular proteostasis [24]. However, it is plausible in these cells that the altered proteostasis is simply a result of the macrophages' stress response. In particular we note an enrichment for proteasome genes involved with the activation of NF-kappaB (NF-kB), a pro-inflammatory transcription factor on which multiple 


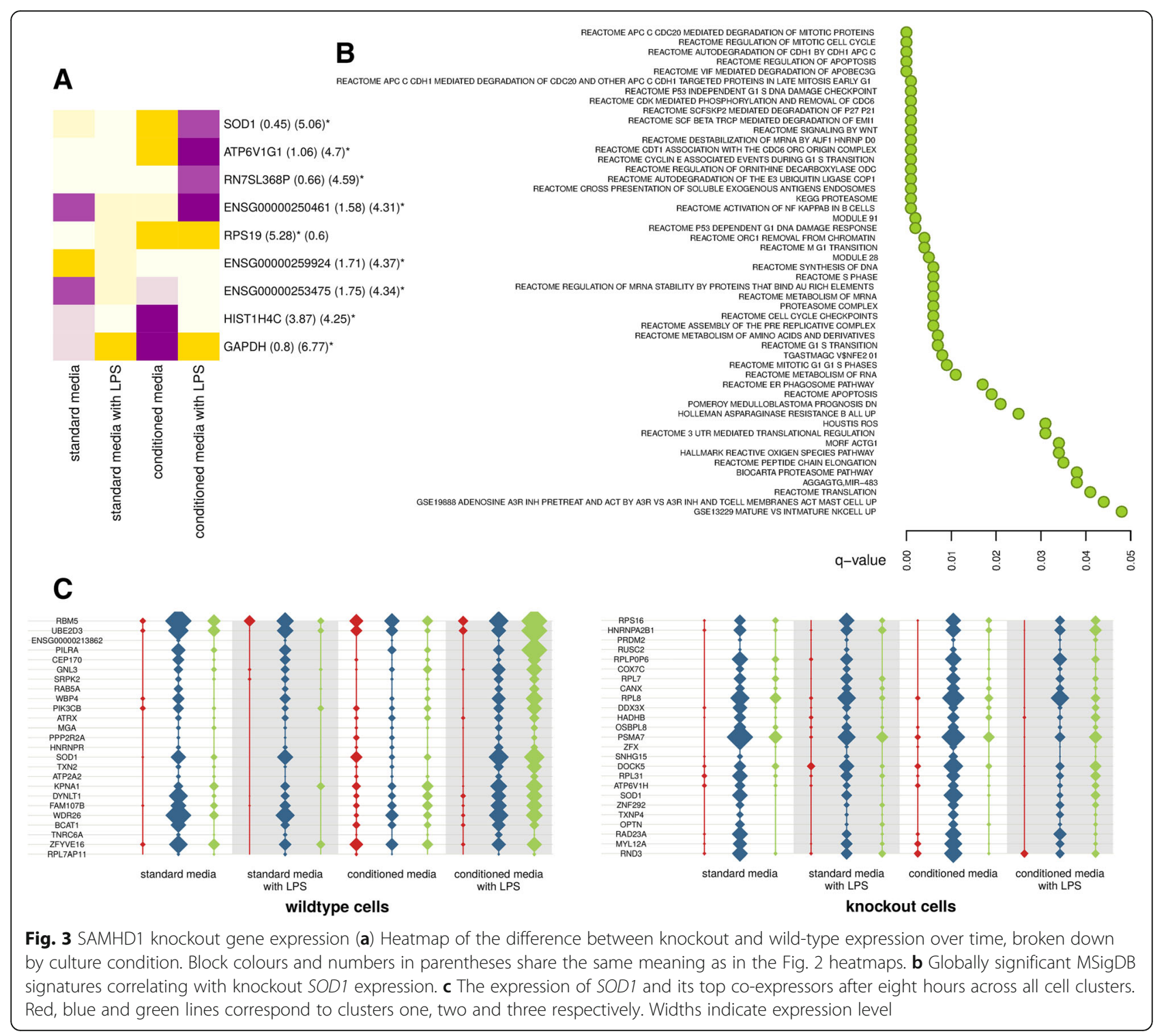

macrophage signaling pathways converge [25]. A similar proteostasis theme is observed for the regulation of ornithine decarboxylase, a macrophage anti-inflammatory enzyme [26] that metabolises a well described marker of macrophage pro- versus anti-inflammatory phenotype polarisation. With these pathway associations validating this approach by supporting known biology of increased inflammation with SAMHD1 knockout - albeit within the context of oxidative stress and altered proteostasis we asked if other pathways in Fig. 3b point to unexpected biological themes.

A striking pathway result was the repeated enrichment for proteasome genes involved in the degradation of cell cycle proteins. While the direct causal relationship between cell cycle and SAMHD1 is considered to be via its cyclin-dependent kinase phosphorylation and inhibition [27], these results point to an additional reverse relationship. A tumor suppressor role for SAMHD1 has been proposed based on its maintenance of genome integrity and cancer associations with downregulation $[12,13]$. However, the pathways in Fig. 3b counter-intuitively point to p53 dependent and independent $G_{1}$ arrest as part of the DNA damage response. These results highlight a necessary fine balance in SAMHD1 activity in terms of cell cycle control, with too little SAMHD1 arresting cell cycle progression via other tumour suppressors in response to DNA damage. This relationship between SAMHD1 and $G_{1}$ arrest has been demonstrated in dividing fibroblasts that turn senescent [28], though an important difference here is that it suggests the wild-type $G_{0}$ (post-mitotic) macrophages shift to a $G_{1}$ block with SAMHD1 knockout. Finally, and perhaps most interesting in terms of the HIV1 host-pathogen relationship, is the highly significant pathway enrichment for the degradation of APOBEC3G 
as part of the altered stress response in SAMHD1 knockouts. This HIV-1 restriction factor causes proviral DNA hypermutation via cytidine deamination, and so shares no known overlap in antiviral activity with SAMHD1 [29].

\section{Discussion and conclusions}

As single-cell methods continue to mature, so will the ability to move beyond simple 'snapshots' of cell populations towards studying the determinants of population dynamics. We expect that one area of demand for this type of single-cell functional genomics will be cellular genetics models, with large-scale efforts already underway to generate stem cell banks to support tissuespecific insights into genetic variants [30,31]. Even with the simplest such models, single-cell sequencing will prove useful to rule out heterogeneity unrelated to the cell phenotypes of interest. In our macrophage model, for example, we have seen that while the majority of cells adopted a tissue-resident phenotype, cells that behave quite differently can be present. As the co-culture and genetic complexity of cellular genetic models increases, so will the demand for methods to confidently map heterogeneity with high replicability across multiple culture or laboratory conditions. Automated and standardized microfluidics present a decided advantage in this regard. In this proof-of-principle work we have been able to generate complex experimental designs within chips over multiple iterations. As all the conditions studied were replicated at least nine times, we have been able to develop and implement statistical models that focus on highly replicated cell behaviors that could easily be integrated with results from other laboratories.

Perhaps the most distinct advantage of such lab-on-chip microfluidics is the ability to perform imaging, temporal and microenvironmental analyses of cell population dynamics. In this study these variables allowed us, for example, to comment on the altered oxidative stress response (SOD1 expression) with SAMHD1 knockout and macrophage signaling. By blocking the signaling component we observed a significant reduction in this knockout effect. Interestingly, SOD1 and SAMHD1 have known respective gain and loss of function associations with neuroinflammation that are in keeping with effects noticed in this model. We speculate that targeting the observed signaling component may provide an avenue for the treatment of these and other neurodegenerative diseases influenced by innate immunity. Microglia (central nervous system macrophages), for example, are not only implicated in HIV associated neurocognitive disease (HAND), but also in Alzheimer's disease via aberrant inflammatory signalling [32]. Future work with the Polaris ${ }^{\mathrm{Tm}}$, assaying single-cell macrophage supernatants, may prove useful in narrowing down this as yet unidentified signaling factor. In this study, single-cell SOD1 coexpression pathway analysis enriched for altered proteostasis. The most widely observed biological association with this altered proteostasis was a cell cycle $\left(\mathrm{G}_{1}\right)$ block as part of the DNA damage response. While an increased dNTP pool with SAMHD1 loss of function is known to reduce genome integrity, the SOD1 association with these pathways point to an oxidative stress contribution to DNA breaks triggering $G_{1}$ checkpoint genes. SOD1 may also directly contribute to this arrest via its anti-apoptotic signaling [33]. Perhaps most intriguing is how these associations could provide insights into activating latent viruses within therapeutically intractable reservoirs such as macrophages and resting CD4+ T cells, where SAMHD1 is highly expressed [34]. Triggering apoptosis signaling has been proposed as one therapeutic strategy for HIV-1 activation [35], which would require SOD1 apoptosis inhibition to be minimised. Our knockout cells strongly suppressed SOD1 expression when activated in conditioned media, suggesting that promoting macrophage activation in combination with SAMHD1 inhibition would, at least in vitro, be the most effective strategy to purge latent viruses. Infection of the macrophages with HIV-1 in these microfluidic chips to directly study these effects is one promising approach to study this. Such HIV-1 infection studies with other macrophage knockouts such as the HIV-1 restriction factor APOBEC3G, may prove particularly enlightening, as results from this study suggest a previously unappreciated connection between SAMHD1 loss of function and APOBEC3G degradation. Understanding the conditions under which SAMHD1 inhibition also results in reduced APOBEC3G levels would be of direct relevance to therapies aimed at viral activation. Under these situations, SAMHD1 inhibition might have the desired activation response, but reduced APOBEC3G would enhance the ability of the activated viruses to reinfect other cells.

\section{Methods}

Stem cells, generation of macrophages and experimental media

The human embryonic stem cell line HUES-2 was obtained from the HUES Facility, University of Harvard [36]. Feeder-free PSC cells were cultured in $\mathrm{mTeSR}^{\mathrm{TM}}-1$ medium (Stem Cell Technologies) on Matrigel (Corning)coated tissue culture dishes, passaged with TrypLE (Invitrogen) with the addition of $10 \mu \mathrm{mol} / \mathrm{L}$ Rho-kinase inhibitor Y-27632 (Abcam). A double-nicking CRISPRCas9 approach was used to generate SAMHD1-knockout stem cell lines [37]. Plasmid pX462 (gift from Feng Zhang; Addgene plasmids cat. 48141, [38]), expressing the guide RNA, D10A-mutated Cas9 and a puromycin-selection cassette was adapted to target $S A M H D 1$ at exon 4 (GTGTATCAATGATTCGGACGAGG and CGATACAT CAAACAGCTGGGAGG; PAM underlined) or exon 5 target sites (CGTTCACTTATCTGCAGCTCTGG and 
GGATGTCTAGTTCACGCACTGGG; PAM underlined) using protocols previously described $[38,39]$. PSCs were transfected with all four plasmids targeting SAMHD1 using the Neon ${ }^{\circ}$ Transfection system (Invitrogen) according to manufacturer's guidelines $\left(2 \times 10^{6}\right.$ cells electroporated with $15 \mu \mathrm{g}$ DNA using a $100 \mu \mathrm{L}$ tip at $1000 \mathrm{~V}, 40 \mathrm{~ms}$ pulse width, one pulse), cultured without antibiotics for $48 \mathrm{~h}$ and then for $48 \mathrm{~h}$ with selection in $0.4 \mu \mathrm{g} / \mathrm{mL}$ puromycin (Sigma). Single-cell clones were generated by plating transfected PSCs at low density onto mitotically-inactivated mouse embryonic feeder (MEF) cells $[40,41]$ on gelatincoated tissue culture plates in stem cell medium (KODMEM, $2 \mathrm{mmol} / \mathrm{L}$ L-Glutamine, $100 \mathrm{mmol} / \mathrm{L}$ nonessential amino acids, 20\% serum replacement, and $8 \mathrm{ng} / \mathrm{mL}$ FGF2; Invitrogen). Clones with modifications at the SAMHD1 locus were identified by high resolution melt analysis. Sequencing confirmed that clone E2 had an out-of-frame insertion (29 and $49 \mathrm{bp}$ ) into each allele of SAMHD1 exon 4 and G9 had an out-of-frame deletion (43 bp) in one allele and an in-frame deletion (39 bp) that deleted the essential allosteric GTP binding site (amino acids 135 to 147) and would alter the catalytic site. Macrophages derived from these clones were screened for SAMHD1 expression by western blotting using a mouse anti-SAMHD1 antibody (clone 2D7, Insight Biotechnology Ltd) and a rabbit antiGAPDH antibody (Sigma) (Additional file 1: Figure S1).

Feeder-free PSC cells were cultured in $\mathrm{mTeSR}^{\mathrm{TM}}-1$ medium (Stem Cell Technologies) on Matrigel (Corning)coated tissue culture dishes, passaged with TrypLE (Invitrogen) with the addition of $10 \mu \mathrm{mol} / \mathrm{L}$ Rho-kinase inhibitor Y-27632 (Abcam). A protocol devised in our laboratory was used to generate macrophages from PSC cultures. Briefly, embryoid bodies were formed using the spin method in AggreWells ${ }^{\text {rm }} 800$ (Stemcell Technologies) plates, each of which was split into two monocyte factories in T175 tissue culture flasks containing 150 embryoid bodies. The monocytes released into the supernatant were harvested regularly and plated into 96-well plates at $5 \times 10^{4}$ cells per well in macrophage differentiation medium consisting of $\mathrm{XVIVO}^{\mathrm{m}} 15$ (Lonza) supplemented with $100 \mathrm{ng} / \mathrm{mL}$ M-CSF (Invitrogen), $2 \mathrm{mM}$ glutamax (Invitrogen), $100 \mathrm{U} / \mathrm{mL}$ penicillin and $100 \mu \mathrm{g} / \mathrm{mL}$ streptomycin (Invitrogen). Four days after plating the media was replaced with fresh macrophage differentiation media with additional 10\% fetal bovine serum (FBS; Invitrogen) and the cells were used on day 7 of differentiation. We used three batches (A, B, G, each corresponding to one AggreWells $\mathrm{s}^{\mathrm{m}} 800$ plate) of wild-type cells and two batches (G9: C, D and E2: E, F) each of the two SAMHD1-knockout clones for the Polaris macrophage stimulation experiments.

Four kinds of media ('conditioned' and 'standard', with or without LPS) were used in the microfluidic chips. To generate conditioned media, stem cell-derived monocytes were plated at $5 \times 10^{5}$ cells/well in a 12-well tissue culture plate and differentiated for 4 days in macrophage differentiation medium followed by 3 days in the presence of $10 \%$ FBS. The medium was then replaced with fresh macrophage medium plus $10 \%$ FBS with or without $100 \mathrm{ng} / \mathrm{mL}$ LPS (Sigma). "Standard media" were generated by incubation of macrophage differentiation medium plus 10\% FBS either with or without $100 \mathrm{ng} / \mathrm{ml} \mathrm{LPS}$ at $37{ }^{\circ} \mathrm{C}$ to simulate incubation of conditioned media with cells. For each kind of medium, after $24 \mathrm{~h}$ with LPS/mock stimulation, supernatants were recovered by centrifugation, $0.45 \mu \mathrm{m}$-filtered and stored at $-80{ }^{\circ} \mathrm{C}$ then thawed and clarified by centrifugation at 14,100 $\mathrm{rcf}$ for $10 \mathrm{~min}$ before use.

\section{Polaris $^{\mathrm{TM}}$ protocols}

Polaris runs followed the protocol 'Using Polaris to Generate Single-Cell cDNA Libraries for mRNA Sequencing' (PN 101-0082 A1, Fluidigm). Set-up parameters are shown in Table S1. Polaris integrated fluidic circuits (IFCs) were prepared for cell capture by a priming step, during which the capture chambers were also coated with the extracellular matrix compound fibronectin (25 ng/ $\mu$ l, cat. F4759, Sigma-Aldrich) for handling adherent cells, and capture beads (prepared to Fluidigm specifications) were loaded to prevent the release of captured single cells (Fig. 1). Priming was arranged to finish as the cell mix became ready for loading (Additional file 1: Figure S2A).

For each experimental run, one well of cells from a single batch each of wild-type and knockout cells was used. One sample was stained with CellTracker ${ }^{\text {mix }}$ Orange CMRA Dye and the other with both CellTracker ${ }^{\text {tx }}$ Orange CMRA and CellTracker ${ }^{\mathrm{ra}}$ Green CMFDA Dye (cat. C34551 and C7025, Thermo Fisher Scientific) in Wash Buffer (Fluidigm). Dye concentrations were adjusted upwards between runs during the experiment to improve sensitivity and resolution: $1 \mu \mathrm{M}$ for runs 1 and 2; $2 \mu \mathrm{M}$ for runs 3 to 12 ; and $3 \mu \mathrm{M}$ for runs 13 to 20 . Wild-type and knockout cells were dual- or singlestained in approximately equal numbers of experiments (Additional file 1: Figure SB). The culture medium was removed and the cells were washed twice with $250 \mu \mathrm{l}$ Wash Buffer (Fluidigm) then stained with $100 \mu \mathrm{l}$ of staining solution at $37{ }^{\circ} \mathrm{C}$ for $15 \mathrm{~min}$ before a further wash with $150 \mu \mathrm{l}$ of Wash Buffer. The Wash Buffer was removed, $100 \mu \mathrm{l}$ of $0.5 \mathrm{mM}$ EDTA (cat. 15575, Gibco) in PBS was added and the cells were incubated at $37{ }^{\circ} \mathrm{C}$ for 15 min before the EDTA was removed and the cells were resuspended gently in $50 \mu \mathrm{l}$ of Feed Media $\left(\mathrm{X}-\mathrm{VIVO}^{\mathrm{Ta}} 15\right.$ cat. BE02-061Q (Lonza), 1\% PenicillinStreptomycin (10,000 U/mL), cat. 15140-122, (Gibco)). Cells were counted on a TC-20TM Automated Cell Counter (Bio-Rad) and samples were adjusted to a final concentration of $350-400$ cells $/ \mu \mathrm{l}$. 
Fluidigm guidelines (Fluidigm Single-Cell Preparation Guide, PN 100-7697) were used to establish optimal buoyancy at an 4:1 (cells:cell suspension reagent) ratio for the Polaris experiment. For each run, $15 \mu \mathrm{l}$ each of differentially stained wild type and knockout cells were mixed with $7.5 \mu \mathrm{l}$ of Cell Suspension Reagent (Fluidigm, PN 101-0434) $25 \mu \mathrm{l}$ of the resulting suspension, containing an estimated 7000-8000 cells, was loaded on the Polaris IFC. On-board imaging settings to control automatic cell selection were set for each run, with a threshold that varied between 4000 and 6000 for a constant $1.0 \mathrm{~s}$ exposure. After completion of the cell selection step, the IFC was removed from the Polaris system and placed in an incubator at $37{ }^{\circ} \mathrm{C}$ and $5 \% \mathrm{CO}_{2}$ for $2 \mathrm{~h}$ to allow the cells to settle prior to dosing. Dosing culture media were centrifuged in small volumes $(500 \mu \mathrm{l})$ at $14,100 \mathrm{rcf}$ for $10 \mathrm{~min}$ before $27 \mu \mathrm{l}$ of medium and $200 \mu \mathrm{l}$ of Feed Media were loaded into the appropriate wells of the IFC (Additional file 1: Figure S2C). For 1-h dosing runs, the dosing step was stopped manually and for 8 -h dosing runs, cells were also dosed at $4 \mathrm{~h}$. Successive runs were scheduled to alternate between one and eight hour dosing.

The Polaris acquired images of all 48 chambers in all available fluorescence channels during cell capture, at the start of dosing and every $1 \mathrm{~h}$ thereafter until the end of the run. IFCs were removed from the Polaris for additional, high-resolution imaging on a Leica TCS SP8 confocal microscope (Leica Microsystems) at 3 stages of each run: after cell capture, after the 2 -h incubation and the end of the dosing step, just before lysis. (Additional file 1: Figure S3A). The imaging protocol was designed to acquire bright-field and fluorescence images, for CellTracker Orange and CellTracker Green: $488 \mathrm{~nm}$ and $561 \mathrm{~nm}$ excitation and hybrid detectors for emission at 500-550 nm and 571-630 nm respectively, were used with a $20 \times$ objective and a template to automatically locate the 48 IFC cell isolation chambers, in an image acquisition process lasting $\sim 5$ min during which the microscope chamber temperature was maintained at $37^{\circ} \mathrm{C}$. The black vinyl film on the lower surface of the Polaris chip was removed temporarily for each imaging stage. For each time-point and channel, the Polaris stored a single image of the IFC's 48 cell chambers, $5200 \times 1000$ pixels in size, in which each pixel had dimensions of approximately $5.5 \times 5.5 \mu \mathrm{m}$. On the Leica, one image of $512 \times 512$ pixels, each $1.39 \times$ 1.39 pixels, was captured per cell chamber in bright field, orange and green channels. The initial aims of the image analysis were to differentiate double- and single-stained cells (wild-type and knockout or vice-versa, depending on the run) and to assess cell shape, motility and phagocytosis behaviour. The $\mathrm{R}$ package EBImage [42] was used with a set of bespoke $\mathrm{R}$ functions to automatically identify individual cell chambers and the cells they carried. Formally, within the large Polaris image(s), cells were detected as clusters of 4 or more of the brightest 30 pixels in the trimmed image corresponding to each cell chamber, for each fluorescent channel. Motility was assessed by measuring changes in the cluster positions between imaging time-points. To assess cell morphology (circularity, the proportion of pixels within the smallest circle encompassing the cluster that belong to the cluster), the higherresolution Leica images were analysed using a re-scaled version of the same algorithm, requiring clusters of 60 or more of the brightest 411 pixels. Automated imaging assessments were checked extensively by eye, leading to the identification of irregularly shaped or unevenly stained cells. Macrophage phagocytosis of capture beads was assessed manually.

For each Polaris run, a bulk control sample of the wildtype and of the knockout cells used in the run was prepared for sequencing. Aliquots containing 2000-4000 of the stained cells loaded on the Polaris were kept at room temperature, incubated along with the loaded IFC for the 2-h incubation step, and kept at $37{ }^{\circ} \mathrm{C}$ during dosing. Within 30 min of starting the Polaris lysis step, bulk-cell samples were lysed and processed using the RNA extraction kit RNeasy Micro Kit (cat\# 74004, Qiagen) and eluted in $14 \mu \mathrm{l}$ of RNAse-free water. At the end of the dosing protocol and after any additional imaging, cells were lysed for reverse transcription and amplification for cDNA generation in the Polaris. Master mixes for this procedure were prepared using the SMARTer Ultra Low RNA Kit (Clontech), according to the Fluidigm Polaris protocol with minor modifications. The cell lysis mix $(28 \mu \mathrm{l})$ contained $8.0 \mu$ l Polaris Lysis Reagent, $9.6 \mu \mathrm{l}$ of a $1 / 200$ dilution of Polaris Lysis Plus Reagent (in PCR-grade water, prepared immediately before use), 9.0 $\mu$ l SMARTer kit 3' SMART CDS Primer IIA and (for runs 1-11) $1.4 \mu \mathrm{l}$ of diluted ERCC spike-in RNA, prepared by adding $1.0 \mu \mathrm{l}$ of a 1/10 dilution of ERCC ExFold RNA Spike-In Mixes (cat. 4456739, Ambion) to $96.5 \mu \mathrm{l}$ Polaris Loading Reagent and $2.5 \mu \mathrm{l}$ SMARTer Kit RNase Inhibitor $(40 \mathrm{U} / \mu \mathrm{l})$. The reverse transcription reaction mix $(48 \mu \mathrm{l})$ contained $15.5 \mu \mathrm{l}$ $5 \times$ First-Strand Buffer (RNase-free), $1.9 \mu \mathrm{l}$ DTT, $7.7 \mu \mathrm{l}$ dNTP mix, $7.7 \mu$ l IIA Oligonucleotide, $1.9 \mu$ l RNAse Inhibitor, $7.7 \mu \mathrm{l}$ SMARTscribe Reverse Transcriptase (all SMARTer Kit, Clontech), 2.4 $\mu$ l Polaris Loading Reagent and $3.2 \mu \mathrm{l}$ Polaris RT Plus Reagent. The PCR mix $(90 \mu \mathrm{l})$ contained $63.5 \mu \mathrm{l}$ PCR-grade water, $10.0 \mu \mathrm{l} 10 \times$ Advantage 2 PCR Buffer (not SA - Short Amplicon), $4.0 \mu \mathrm{l} 50 \times$ dNTP Mix, 4.0 $\mu$ l 50× Advantage 2 Polymerase Mix (all Advantage 2 Kit, Clontech) and $4.0 \mu$ SMARTer IS PCR primer. The cDNA products were harvested into a 96well plate in the arrangement shown in Additional file 1: Figure S2D. Harvest wells with atypical volumes (some with no material, others with an excess) were excluded from further analysis. Bulk control samples comprising $1 \mu \mathrm{l}$ of RNA extracted from the bulk samples above were 
each mixed with $4.5 \mu \mathrm{l}$ of cell lysis mix and processed using the following temperature sequence: $37{ }^{\circ} \mathrm{C}$ for $5 \mathrm{~min}, 72{ }^{\circ} \mathrm{C}$ for $3 \mathrm{~min}, 25^{\circ} \mathrm{C}$ for $1 \mathrm{~min}$, then $4{ }^{\circ} \mathrm{C}$ (hold), then reverse transcribed using $9.0 \mu \mathrm{l}$ of the reaction mix, at $42{ }^{\circ} \mathrm{C}$ for $90 \mathrm{~min}$ followed by enzyme inactivation at $70{ }^{\circ} \mathrm{C}$ for $10 \mathrm{~min}$, then $4{ }^{\circ} \mathrm{C}$ (hold). Bulk-sample PCRs contained $1 \mu \mathrm{l}$ of cDNA generated in the previous step and $9.3 \mu \mathrm{l} \mathrm{PCR} \mathrm{mix.} \mathrm{PCR} \mathrm{conditions} \mathrm{were} \mathrm{as} \mathrm{follows:} 95^{\circ} \mathrm{C}$ for 1 min then 5 cycles of $\left\{95^{\circ} \mathrm{C} / 20 \mathrm{~s}, 58{ }^{\circ} \mathrm{C} / 4 \mathrm{~min}, 68^{\circ} \mathrm{C} /\right.$ $6 \mathrm{~min}\}, 9$ cycles of $\left\{95^{\circ} \mathrm{C} / 20 \mathrm{~s}, 64{ }^{\circ} \mathrm{C} / 30 \mathrm{~s}, 68^{\circ} \mathrm{C} / 6 \mathrm{~min}\right\}$, 7 cycles of $\left\{95{ }^{\circ} \mathrm{C} / 30 \mathrm{~s}, 64{ }^{\circ} \mathrm{C} / 30 \mathrm{~s}, 68{ }^{\circ} \mathrm{C} / 7 \mathrm{~min}\right\}$, then $72{ }^{\circ} \mathrm{C}$ for $10 \mathrm{~min}$ and $4{ }^{\circ} \mathrm{C}$ (hold). After Quant-iT PicoGreen (Thermo Fisher) normalization of cDNAs to $0.22 \mathrm{ng} / \mu \mathrm{l}$, Illumina sequencing libraries were prepared together using Nextera XT DNA Library Preparation Kit (Illumina), according to the manufacturer's specifications, at quarter-scale on a Beckman-Coulter FXp automated liquid handling instrument, and pooled as up to 192 multiplexed libraries using in-house dual-indexing library tags. Some samples that had failed because of empty cell chambers or the presence of more than one cell in a chamber were included, and two bulk-cell samples per run were included in each pool, which was sequenced as a single lane of $75 \mathrm{~b}$ paired-end reads on an Illumina HiSeq 4000 instrument in 4 different runs.

\section{RNA-Seq data generation and initial quality control}

Samples were prepared and paired-end sequenced using the Illumina HiSeq ${ }^{\text {tm }} 4000$ Sequencing platform, as described above. RNA-seq reads were trimmed for Nextera/ Illumina adapter sequences using skewer-v0.1.125 [43]. Trimmed reads were mapped to a modified reference genome comprising the human genome, Homo sapiens GRCh37, and fasta sequences for ERCC spike-ins (ThermoFisher). Reads in gzipped fastq format were aligned using Hisat2 version-2.0.0-beta [44] with default parameters. Duplicate reads were marked using MarkDuplicates.jar implemented in Picard tools v1.92. BAM alignments were name sorted with Samtools version 1.1. Alignment metrics were calculated using CollectRnaSeqMetrics.jar implemented in Picard tools v1.92 for full BAM files and with potential PCR duplicates marked. RNA-SeQC [45] was used to calculate sequencing bias, as the median estimators in Picard can result in zero estimates. Reads mapping uniquely to genes annotated in ENSEMBL release 76 were counted using featureCounts [46] implemented in subread-v1.5.0 [47]. Read distribution between various features - assigned reads (mapped uniquely to exons), multiple mapping, ambiguous mapping, No features (mapped uniquely to intronic and intergenic regions) - was obtained from featureCounts results. Read counts were normalized to Transcripts per million (TPM), and number of detected genes per sample were calculated by counting genes with at least 1 TPM. Details of the supplied data and meta-data provided are provided in Additional file 1, including further QC using the R scater package [48]. In brief, we excluded culture chambers with visually confirmed doublets (two cells), numbers of detected genes more similar to bulk controls, and cells with very low starting cDNA. FASTQ files for the data have been uploaded to the Gene Expression Omnibus (GSE87849).

\section{Clustering the macrophages and gene expression modeling} All modeling and analyses were performed in the R environment (version 3.2.1, x86_64-pc-linux-gnu 64-bit, Ubuntu 14.04.2 LTS). Multidimensional scaling (MDS) on the cell rank correlations - without gene weighting was used to reduce one hour and eight hour cells to five dimensions, as detailed in the provided Additional file 1 code wrapped in the cellStates() function. Inclusion of a greater number of dimensions did not influence the clustering. Additional file 1: Figure S16 \& S17 demonstrate the cell densities and individual cells for the five dimensions in the eight hour cells, where some clusters of cells can be seen to be unique to individual chips. To ensure reproducibility, the constraint was added that a cluster with fewer than three cells in more than half of the replicates not be considered in the downstream modelling. While such groups of cells may represent illuminating features in the macrophage model's behaviour, these are not reproducible effects.

Extracting major cell states was performed using a hybrid of model-driven clustering (Gaussian mixtures) and non-parametric clustering (partitioning around medoids), as detailed in the Additional file 1 code wrapped in the consensusCluster() function. Repeated fitting of mixtures to the data - with one chip replicate omitted per iteration - was used to produce a consensus matrix of the proportion of iterations cells share clusters. This was nonparametrically re-clustered, selecting the maximum number of clusters for which experimental replication was strongly represented in each cluster. We note that although this combination of methods was employed, other tested approaches such as hierarchically clustering the consensus matrix produced similar results due to the consensus and reproducibility constraints. The defined clusters are recorded in the cell meta-data (Additional file 2). The major latent cell states identified with exploratory data analysis remain the only two identified major states in the one hour cells, with a third reproducible cluster emerging at eight hours that shares properties with the main group of cells (Additional file 1: Figure S18).

Altered gene expression was modelled as the change in mean conditioned on (i.e. tested per) cell subtype of interest. Overall mean across cell subtypes $(\mu)$ was used as a measure of global shift in gene expression, while mean absolute deviation (MAD) of the subtypes was used as a 
measure of variability and so context/subtype specificity. For example, for $d_{i}=$ change in mean expression over time for cell subtype $i, \mu=\left(\mathrm{d}_{1}+\mathrm{d}_{2}+. .+\mathrm{d}_{\mathrm{n}}\right) / \mathrm{n}$ and MAD $=\left(\mid \mathrm{d}_{1}-\right.$ $\left.\mu|+| d_{2}-\mu|+. .+| d_{n}-\mu \mid\right) / n$. More robust estimators, such as the use of quantiles, provided similar top hits, so here we present $\mu$ and MAD estimates, focusing rather on a rank product framework to determine statistical significance of rank reproducibility per sequencing library [15]. A focus on gene ranks has several advantages well suited to single-cell work: it is non-parametric, robust, comments directly on reproducibility, and allows data fusion or meta-analysis without the need for complex data normalisations. For computational speed, the Heskes rank product algorithm was used to assess bounds on the statistical significance. Reported p-values are the geometric means of the upper and lower bounds provided by the rankprodbounds() function, with the qvalue package used to estimate global significance at $5 \%$ false discovery rate [49]. Rank (Spearman) correlations were used to estimate gene co-expression, followed by testing for co-expression signature enrichment with Preranked Gene Set Enrichment Analysis (default settings) [23].

\section{Additional files}

\section{Additional file 1: Supplementary Methods, Figures and Tables. (DOCX $3991 \mathrm{~kb}$ )}

Additional file 2: Supplementary Data. (XLSX 30285 kb)

\section{Abbreviations}

HAND: HIV associated neurocognitive disease; LPS: Lipopolysaccharide; PSC: Pluripotent stem cells; ROS: Reactive oxygen species

\section{Acknowledgements}

We thank the WTCHG Cellular Imaging, High Throughput Genomics, and Bioinformatics and Statistical Genetics cores for their support. We also thank Zam Cader and Tudor Fulga for their critical reading of the manuscript.

\section{Funding}

This study, within the Oxford Single-cell Biology Consortium, was funded by a core Wellcome Trust award at the WTCHG (grant 090532/Z/09/Z), in addition to contributions from the UK Biotechnology and Biological Sciences Research Council (BBSRC) and the Oxford University John Fell Fund. RN and DW are funded by Wellcome Trust and BBSRC studentships respectively. SPP's research group is funded by the Nuffield Department of Medicine Leadership Fellowship.

\section{Availability of data and material}

All data generated or analysed during this study are included in this published article and its supplementary information files.

\section{Authors' contributions}

QW, MM and RB conceived and supervised the study. DW and MM created and provided the cell model. EM, ML, MG, JW and AL implemented the microfluidics platform. EM optimised and ran the experimental work. RN, QW and SP provided imaging analysis. JB, ES, BW and HL provided RNA sequencing core facility support. QW provided the experimental design and data modeling. SF contributed to the clustering analysis. CH provided additional statistical support. QW and RB wrote the manuscript. WJ provided critical reading and guidance for the manuscript. All authors read and approved the final manuscript.

\section{Competing interests}

$M L, M G, J W$, and $A L$ are employees of Fluidigm Corporation.

\section{Consent for publication}

Not applicable.

\section{Ethics approval and consent to participate}

Work on human stem cell lines was reviewed and approved by the UK Stem Cell Bank Steering Committee (Medical Research Council, London UK, 20.10.2005).

\section{Author details}

${ }^{1}$ Wellcome Trust Centre for Human Genetics (WTCHG), University of Oxford, Oxford OX3 7BN, UK. ${ }^{2}$ Weatherall Institute of Molecular Medicine (WIMM), University of Oxford, Oxford OX3 9DS, UK. ${ }^{3}$ Division of Structural Biology, University of Oxford, Oxford OX3 7BN, UK. ${ }^{4}$ Sir William Dunn School of Pathology, University of Oxford, Oxford OX1 3RE, UK. ${ }^{5}$ Fluidigm Corporation, 7000 Shoreline Ct Ste 100, South San Francisco, CA 94080-7603, USA.

${ }^{6}$ Department of Statistics, University of Oxford, Oxford OX3 3LB, UK.

Received: 27 August 2016 Accepted: 20 December 2016

Published online: 07 January 2017

\section{References}

1. Martinez FO, Gordon S. The M1 and M2 paradigm of macrophage activation: time for reassessment. F1000Prime Rep. 2014;6:13.

2. Barton K, Winckelmann A, Palmer S. HIV-1 Reservoirs During Suppressive Therapy. Trends Microbiol. 2016;24:345-55.

3. Rappaport J, Volsky DJ. Role of the macrophage in HIV-associated neurocognitive disorders and other comorbidities in patients on effective antiretroviral treatment. J Neurovirol. 2015;21:235-41.

4. van Wilgenburg $B$, et al. Efficient, long term production of monocytederived macrophages from human pluripotent stem cells under partlydefined and fully-defined conditions. PLoS One. 2013;8:e71098.

5. Flynn R, Grundmann A, Renz P, Hänseler W, James WS, Cowley SA, et al. CRISPR-mediated genotypic and phenotypic correction of a chronic granulomatous disease mutation in human iPS cells. Exp Hematol. 2015; 43:838-48.

6. Fluidigm. https://www.fluidigm.com/products/polaris. Accessed 1 Oct 2016.

7. Alasoo K, Martinez FO, Hale C, Gordon S, Powrie F, Dougan G, et al. Transcriptional profiling of macrophages derived from monocytes and iPS cells identifies a conserved response to LPS and novel alternative transcription. Sci Rep. 2015;5:12524.

8. Karlsson KRE, et al. Homogeneous monocytes and macrophages from human embryonic stem cells following coculture-free differentiation in M-CSF and IL-3. Exp Hematol. 2008;36:1167-75.

9. Vanhee S, De Mulder K, Van Caeneghem Y, Verstichel G, Van Roy N, Menten $\mathrm{B}$, et al. In vitro human embryonic stem cell hematopoiesis mimics MYB-independent yolk sac hematopoiesis. Haematologica. 2015;100:157-66.

10. Sattentau QJ, Stevenson M. Macrophages and HIV-1: An Unhealthy Constellation. Cell Host Microbe. 2016:19:304-10.

11. Simon V, Bloch N, Landau NR. Intrinsic host restrictions to HIV-1 and mechanisms of viral escape. Nat Immunol. 2015;16:546-53.

12. Kohnken R, Kodigepalli KM, Wu L. Regulation of deoxynucleotide metabolism in cancer: novel mechanisms and therapeutic implications. Mol Cancer. 2015;14:176.

13. Clifford R, Louis T, Robbe P, Ackroyd S, Burns A, Timbs AT, et al. SAMHD1 is mutated recurrently in chronic lymphocytic leukemia and is involved in response to DNA damage. Blood. 2014;123:1021-31.

14. Crow YJ, Rehwinkel J. Aicardi-Goutieres syndrome and related phenotypes: linking nucleic acid metabolism with autoimmunity. Hum Mol Genet. 2009:18:R130-6.

15. Heskes T, Eisinga R, Breitling R. A fast algorithm for determining bounds and accurate approximate $\mathrm{p}$-values of the rank product statistic for replicate experiments. BMC Bioinformatics. 2014;15:367.

16. Mertens MT, Singh JA. Anakinra for rheumatoid arthritis. Cochrane Database Syst Rev. 2009;21:CD005121.

17. Rodríguez-Prados J-C, Través PG, Cuenca J, Rico D, Aragonés J, Martín-Sanz P, et al. Substrate fate in activated macrophages: a comparison between innate, classic, and alternative activation. J Immunol. 2010;185:605-14. 
18. Shi C, Sakuma M, Mooroka T, Liscoe A, Gao H, Croce KJ, et al. Downregulation of the forkhead transcription factor Foxp1 is required for monocyte differentiation and macrophage function. Blood. 2008;112:4699-711.

19. Gomez JL, Crisafi GM, Holm CT, Meyers DA, Hawkins GA, Bleecker ER, et al. Genetic variation in chitinase 3-like 1 (CHI3L1) contributes to asthma severity and airway expression of YKL-40. J Allergy Clin Immunol. 2015;136:51-8.

20. Geng J, Sun X, Wang P, Zhang S, Wang X, Wu H, et al. Kinases Mst1 and Mst2 positively regulate phagocytic induction of reactive oxygen species and bactericidal activity. Nat Immunol. 2015;16:1142-52.

21. Agrawal L, Louboutin J-P, Reyes BAS, Van Bockstaele EJ, Strayer DS. Antioxidant enzyme gene delivery to protect from HIV-1 gp120-induced neuronal apoptosis. Gene Ther. 2006;13:1645-56.

22. Rouleau G, Guy R. Mutations in Cu/Zn superoxide dismutase gene are associated with familial amyotrophic lateral sclerosis. Nature. 1993;362:59-62.

23. Subramanian A, Tamayo P, Mootha VK, Mukherjee S, Ebert BL, Gillette MA, et al. Gene set enrichment analysis: a knowledge-based approach for interpreting genome-wide expression profiles. Proc Natl Acad Sci U S A. 2005;102:15545-50.

24. Kitamura A, Inada N, Kubota H, Matsumoto G, Kinjo M, Morimoto Rl, et al. Dysregulation of the proteasome increases the toxicity of ALS-linked mutant SOD1. Genes Cells. 2014;19:209-24.

25. Wang $\mathrm{N}$, Liang $\mathrm{H}$, Zen K. Molecular mechanisms that influence the macrophage m1-m2 polarization balance. Front Immunol. 2014;5:614.

26. Liao Y-F, Ya-Fan L, Hui-Chih H, Pei-Chen H, Ming-Ching K, Tzyh-Chyuan H, et al. Ornithine decarboxylase interferes with macrophage-like differentiation and matrix metalloproteinase- 9 expression by tumor necrosis factor alpha via NF-kB. Leuk Res. 2008:32:1124-40.

27. Cribier A, Descours B, Valadão ALC, Laguette N, Benkirane M. Phosphorylation of SAMHD1 by cyclin A2/CDK1 regulates its restriction activity toward HIV-1. Cell Rep. 2013;3:1036-43.

28. Kretschmer S, Wolf C, König N, Staroske W, Guck J, Häusler M, et al. SAMHD1 prevents autoimmunity by maintaining genome stability. Ann Rheum Dis. 2015;74:e17.

29. Strebel K, Klaus S. HIV accessory proteins versus host restriction factors. Curr Opin Virol. 2013:3:692-9.

30. Hipsci [Internet]. 2016. Available from: http://www.hipsci.org/. Cited 24 Jul 2016.

31. StemBANCC. http://stembancc.org. Accessed 1 Oct 2016.

32. Heneka MT, Golenbock DT, Latz E. Innate immunity in Alzheimer's disease. Nat Immunol. 2015;16:229-36.

33. Papa L, Manfredi G, Germain D. SOD1, an unexpected novel target for cancer therapy. Genes Cancer. 2014;5:15-21.

34. Baldauf H-M, Hanna-Mari B, Xiaoyu P, Elina E, Sarah S, Waaqo D, et al. SAMHD1 restricts HIV-1 infection in resting CD4 T cells. Nat Med. 2012;18:1682-9.

35. Khan SZ, Hand N, Zeichner SL. Apoptosis-induced activation of HIV-1 in latently infected cell lines. Retrovirology. 2015;12:42.

36. Cowan CA, Klimanskaya I, McMahon J, Atienza J, Witmyer J, Zucker JP, et al. Derivation of embryonic stem-cell lines from human blastocysts. N Engl J Med. 2004;350:1353-6.

37. Ran FA, Hsu PD, Lin C-Y, Gootenberg JS, Konermann S, Trevino AE, et al. Double nicking by RNA-guided CRISPR Cas9 for enhanced genome editing specificity. Cell. 2013;154:1380-9.

38. Ran FA, Ann Ran F, Hsu PD, Jason W, Vineeta A, Scott DA, et al. Genome engineering using the CRISPR-Cas9 system. Nat Protoc. 2013;8:2281-308.

39. Cong L, Ran FA, Cox D, Lin S, Barretto R, Habib N, et al. Multiplex Genome Engineering Using CRISPR/Cas Systems. Science. 2013;339:819-23.

40. Chia R, Achilli F, Festing MFW, Fisher EMC. The origins and uses of mouse outbred stocks. Nat Genet. 2005;37:1181-6.

41. Gardner RL. Investigation of cell lineage and differentiation in the extraembryonic endoderm of the mouse embryo. J Embryol Exp Morphol. 1982;68:175-98.

42. Pau G, Fuchs F, Sklyar O, Boutros M, Huber W. EBlmage-an R package for image processing with applications to cellular phenotypes. Bioinformatics. 2010;26:979-81.

43. Jiang $H$, Lei R, Ding S-W, Zhu S. Skewer: a fast and accurate adapter trimmer for next-generation sequencing paired-end reads. BMC Bioinformatics. 2014;15:182.

44. Kim D, Langmead B, Salzberg SL. HISAT: a fast spliced aligner with low memory requirements. Nat Methods. 2015;12:357-60.
45. DeLuca DS, Levin JZ, Sivachenko A, Fennell T, Nazaire M-D, Williams C, et al. RNA-SeQC: RNA-seq metrics for quality control and process optimization. Bioinformatics. 2012;28:1530-2.

46. Liao Y, Smyth GK, Shi W. featureCounts: an efficient general purpose program for assigning sequence reads to genomic features. Bioinformatics. 2014;30:923-30

47. Liao Y, Smyth GK, Shi W. The Subread aligner: fast, accurate and scalable read mapping by seed-and-vote. Nucleic Acids Res. 2013;41:e108.

48. scater. http://bioconductor.org/packages/scater. Accessed 1 Oct 2016.

49. Liang K, Kun L, Dan N. Adaptive and dynamic adaptive procedures for false discovery rate control and estimation. J R Stat Soc Series B Stat Methodol. $2011 ; 74: 163-82$

\section{Submit your next manuscript to BioMed Central and we will help you at every step:}

- We accept pre-submission inquiries

- Our selector tool helps you to find the most relevant journal

- We provide round the clock customer support

- Convenient online submission

- Thorough peer review

- Inclusion in PubMed and all major indexing services

- Maximum visibility for your research

Submit your manuscript at www.biomedcentral.com/submit
Biomed Central 